\title{
A Qualitative Analysis of the Effects of Task Complexity on the Functional Role of Information
}

\author{
Bogeum Choi and Jaime Arguello \\ School of Information and Library Science, University of North Carolina at Chapel Hill \\ [bochoi,jarguello]@unc.edu
}

\begin{abstract}
An important question in interactive information retrieval (IIR) is: How do task characteristics influence users' needs? In this paper, we investigate the effects of cognitive task complexity on the types of information considered useful for a task. We characterize information types from two perspectives. From one perspective, we classify task-related information items based on inherent characteristics (referred to as info-types): factual statements, concepts/definitions, opinionated statements, and insights-tips/advice related to the task domain. From a second perspective, we used Byström and Järvelin's framework [5] to define information types based on how the information might be used to complete the task (referred to as functional roles): (1) to help the task doer understand the task requirements (problem information); (2) to help the task doer strategize on how to approach the task (problem-solving information); and (3) to help the task doer learn about the task domain (domain information). Our results suggest that: (1) cognitive task complexity influences the functional roles of information items deemed useful for the task (RQ1); (2) certain info-types are more (or less) likely to play certain functional roles (RQ2); and task complexity influences the variety of functional roles played by info-types (RQ3).

ACM Reference Format:

Bogeum Choi and Jaime Arguello. 2020. A Qualitative Analysis of the Effects of Task Complexity on the Functional Role of Information. In 2020 Conference on Human Information Interaction and Retrieval (CHIIR '20), March 14-18, 2020, Vancouver, BC, Canada. ACM, New York, NY, USA, 5 pages. https: //doi.org/10.1145/3343413.3377992
\end{abstract}

\section{INTRODUCTION}

An important question in interactive information retrieval (IIR) is: How do task characteristics influence users' information needs and behaviors? Studying task characteristics can help us understand why people seek information, what types of information they seek, and how the information is used [15]. In this study, we focus on the effect of cognitive task complexity on the types of information considered useful for the task. Task complexity has been viewed from two perspectives: objective and subjective [6]. Objective task complexity is a characteristic of the task itself (independent of the task doer). One influential perspective of objective task complexity is through the lens of cognitive complexity, which relates to the types of mental processes required by the task $[11,12]$. A simple task may require

Permission to make digital or hard copies of all or part of this work for personal or classroom use is granted without fee provided that copies are not made or distributed for profit or commercial advantage and that copies bear this notice and the full citation on the first page. Copyrights for components of this work owned by others than ACM must be honored. Abstracting with credit is permitted. To copy otherwise, or republish to post on servers or to redistribute to lists, requires prior specific permission and/or a fee. Request permissions from permissions@acm.org.

CHIIR '20, March 14-18, 2020, Vancouver, BC, Canada

(C) 2020 Association for Computing Machinery.

ACM ISBN 978-1-4503-6892-6/20/03 . \$15.00

https://doi.org/10.1145/3343413.3377992 rote memorization, while a complex task may require evaluating different alternatives. Byström and Järvelin $[3,5]$ provided a useful conceptualization of subjective task complexity. They operationalized task complexity on the basis of a priori determinability-the extent to which a worker can deduce the task requirements, form of the solution, and processes involved. In their studies, task complexity was determined based on participants' perceived determinability. In our research, we investigate objective cognitive complexity.

Information can be characterized along many different dimensions (e.g., objective vs. subjective, concrete vs. abstract, declarative vs. procedural, etc.) In this paper, we characterized information items from two perspectives. From one perspective, we characterize information based on inherent characteristics: (1) facts, (2) concepts/definitions, (3) opinions, and (4) insights-advice related to the task domain. We refer to these four categories as info-types. From a second perspective, we characterize information based on how it might be useful to someone working on the task. To this end, we adopted the framework from Byström and Järvelin [3, 5]. Using this framework, we characterized information as: (1) problem information (PI), which helps the task doer understand the task requirements and structure; (2) problem-solving information (PSI), which helps the task doer strategize on how to approach the task; and (3) domain information (DI), which helps the task doer learn about the task domain. We refer to these three categories as functional roles. While info-types relate to inherent characteristics, functional roles describe how the information may be useful.

In this paper, we present a secondary analysis of data gathered for a previous study [9]. In the parent study, we explored the effects of task complexity on the info-types (i.e., facts, opinions, concepts, and insights) commonly used by participants during a task. Our results found that facts were used more during simple tasks, concepts/definitions were used more during moderately complex tasks, and opinions/insights were used more during highly complex tasks. In other words, during complex (vs. simple) tasks, participants had different needs, which were addressed by different info-types.

In this secondary analysis, we aimed to address the following remaining questions. First, how does task complexity influence the functional role of information considered useful for the task? For example, using the framework from Byström and Järvelin [3, 5], do complex tasks require more PI (to help the task doer decipher the task requirements) and more PSI (to help the task doer decide how to approach the task)? Second, are certain info-types more (or less) likely to play a certain functional role? For example, are task-related facts mostly used as DI, concepts mostly used as PI, and opinions/insights mostly used as PSI? Finally, does task complexity influence the relationship between info-types and their functional roles during a task? For example, as task complexity increases, do info-types play a wider variety of functional roles? 
In the parent study, participants were assigned tasks of varying levels of cognitive complexity and were asked to search for information using a custom-built search system. As the main outcome of the task, participants were asked to construct a written response based on the information found. During the search session, task-related info-types were displayed in a peripheral search assistance tool presented alongside the search results. The info-types displayed were manually curated by three graduate students in Information Science prior to the study. During this preliminary data curation phase, the three graduate students were asked to search for facts, concepts, opinions, and insights that would help someone working on the same task. Additionally, they were asked to provide a justification about how each information item might be useful.

In this paper, we conducted a qualitative analysis of these infotypes gathered. Using the information item and justification, infotypes were assigned to three functional roles: problem information (PI), problem-solving information (PSI), and domain information (DI). We investigate the following three research questions:

RQ1: How does cognitive task complexity influence the functional roles of information items deemed useful for the task?

RQ2: What is the association between info-types and functional roles? In other words, are certain info-types more (or less) likely to play a certain role?

RQ3: How does cognitive task complexity influence the association between info-types and functional roles? For example, do the functional roles of info-types become more (or less) diversified as task complexity increases?

\section{BACKGROUND}

Our research builds on prior studies on the effects of task complexity on searchers' needs and behaviors. Task complexity has been studied from different perspectives. Campbell [6] defined complex tasks as having: (1) multiple outcomes, (2) multiple paths to each outcome, (3) greater uncertainty about the paths to an outcome, and (4) greater interdependence between paths. More closely related to our work, task complexity has also been studied from the perspective of cognitive complexity [7, 12], which relates to the types (and variety) of mental processes required by the task. Jansen et al. [11] (and later Kelly et al. [12]) used Anderson and Krathwohl's taxonomy of learning [1] to create search tasks of varying complexity levels. Studies have found that more cognitively complex tasks are perceived to be more difficult and require more search activity [7, 11, 12, 17].

Another conceptualization of task complexity is through the lens of a priori determinability, which refers to the degree of uncertainty about the task requirements, processes involved, and form of the solution [2-5]. Byström and Järvelin [5] used a priori determinability to categorize tasks into five complexity levels. To illustrate, automatic information processing tasks (the simplest) are completely determinable in terms of the task requirements, processes, and form of the solution. Conversely, genuine decision tasks (the most complex) are completely unstructured-none of the task components (inputs, processes, and outcomes) can be determined in advance.

Byström and Järvelin [5] investigated the effects of $a$ priori determinability on the types of information used by workers in a professional setting. Information types where defined based on functional role: (1) problem information (PI) helps the task doer understand the structure and requirements of the problem; (2) problem-solving information (PSI) helps the task doer strategize on how to approach the problem; and (3) domain information (DI) consists of facts, concepts, and theories in the domain of the problem. To elaborate, PI helps the task doer understand the problem and PSI helps the task doer solve the problem (i.e., determine what PI and DI to use and how). In their study, results found differences in the functional role of information sought and used during simple versus complex tasks. Simple tasks required mostly PI and fact-oriented sources to support rule-based processing. Conversely, complex tasks required all three types of information, particularly more PSI to help with problem-solving. Additionally, complex tasks had greater use of human experts as information sources.

In a follow-up study, Byström [3] examined the effects of $a$ priori determinability on participants' use of three different information types: (1) task information (TI) covers case-specific aspects of the task (only applicable to the task at hand); (2) domain information (DI) consists of information related to the task domain (generally applicable to tasks of the same type); and (3) task-solving information (TSI) provides guidance on what TI and DI to use and how. Consistent with Byström and Järvelin [5], results found that complex tasks required multiple types of information (particularly more TSI) and had greater use of human experts as information sources.

Saastamoinen [14] investigated the effects of task complexity on participants' use of different types of information. Task complexity was determined based on participants' perceptions and information types were categorized as: (1) known items, (2) isolated facts, and (3) information aggregates. Results found that more complex tasks required more information aggregates (broadly applicable) and fewer isolated facts (only applicable to the task at hand).

\section{METHOD}

To investigate RQ1-RQ3, we performed a qualitative analysis of data gathered during a previous study [9]. This parent study investigated the influence of cognitive task complexity on the info-types used by participants to complete tasks. Thirty-two participants each completed the same four tasks of varying levels of cognitive complexity (described below). During each task, participants were asked to search for information using a custom-built search system and construct a written response. Additionally, participants had access to four different types of information (i.e., info-types) in a peripheral search assistance tool referred to as the InfoBoxes (IB). The IB was displayed alongside the search results and presented items of each info-type in different tabs.

Info-types: Our four info-types were defined as follows. Facts were defined as objective and verifiable statements related to the task domain. Facts included declarative statements, statistics, and descriptions. Concepts were defined as noun-phrases representing important principles, ideas, or entities related to the task domain. Opinions were defined as subjective/normative statements related to the task domain. Opinions included views, perspectives, judgements, and evaluations. Finally, insights were defined as tips, advice, or recommendations that would be useful to someone working on the task. Our motivation for studying the use of facts, concepts, opinions, and insights originated from prior work. Crescenzi et al. [10] studied participants' note-taking behaviors during exploratory search tasks. A qualitative analysis of participants' notes found that 
they often included factual statements, concepts/definitions, opinions, and meta-level statements about the task itself-tips/advice, clarifications, and nuances about the task domain.

Info-type Data Collection: In the parent study, the info-types available to participants during each task were manually curated. During a preliminary data collection phase, three graduate students in Information Science (referred to as annotators) were assigned our four tasks and were asked to gather facts, concepts, opinions, and insights that might help someone working on the same task. For each task, each annotator was asked to gather at least 8 items per info-type. Additionally, annotators were asked to provide a justification for how each item might be useful.

Functional Role Annotation: In this paper, we present a qualitative analysis of the info-types gathered by annotators during the preliminary data collection phase described above. To this end, we adopted Bystrom and Jarvelin's framework [3, 5] and manually assigned info-types to three functional roles defined as follows. Problem information (PI) is defined as information that helps the task doer understand the requirements and structure of the task. Problem-solving information (PSI) is defined as information that helps the task doer strategize on how to approach the task. Domain information (DI) is defined as general information about the task domain or information that can be used directly as part of the task's solution. These three functional roles were not mutually exclusive-information items (particularly those consisting of multiple statements) could be assigned to multiple functional roles (e.g., PI and PSI). Info-types were assigned to functional roles by examining the information item itself and the provided justification for how it might be useful to someone else.

To test the reliability of the three functional role definitions, both authors of this paper coded all info-types gathered by one of the annotators (i.e., $1 / 3$ of the data). Based on Cohen's Kappa, the level of agreement was PI $=0.61$, PSI $=0.76$, and DI $=0.69$. All three agreement values were at the level of "substantial" (> .60) [13]. Given this satisfactory level of agreement, one of the authors coded the remaining $2 / 3$ of the data.

Cognitive Complexity Levels: Annotators gathered info-types for four tasks of varying levels of cognitive complexity. Cognitive task complexity relates to the types (and variety) of mental processes required by the task. This characterization of task complexity originated from educational research. Anderson and Krathwohl's two-dimensional taxonomy of learning was designed to more precisely define learning objectives for students [1]. In the context of IIR, Jansen et al. [11] (and later Kelly et al. [12]) adopted the cognitive process dimension of A\&K's taxonomy to design learningoriented search tasks of varying complexity levels. In this work, we focused on four complexity levels: remember, understand, analyze, and create. A remember task requires finding or verifying a specific piece of information. An understand task requires engaging with information more deeply in order to exemplify, summarize, and/or explain. An analyze task requires identifying alternatives and understanding their similarities and differences. Finally, a create task requires generating a novel solution to a problem. Our four tasks were adapted from tasks used in Kelly et al. [12] and Capra et al. [7]

To illustrate, our analyze task was as follows: "You are planning an extended hiking trip and will not be able to carry all the water you will need. There will be streams near where you are hiking, but a friend said that you might get sick from drinking water directly from the streams. What are some different methods to purify stream water for drinking during long hiking trips and how do they differ?"

Our remember task required participants to determine how many people in the U.S. are living with HIV. Our understand task required participants to identify human activities that degrade soil quality and provide a brief explanation. Our create task required participants to generate a plan for designing a Soapbox Derby car.

\section{RESULTS}

RQ1. Effects of task complexity on functional roles of taskrelated information: Figure 1 shows the effects of task complexity on the functional roles associated with all info-types combined. In Figures 1-3, the percentages are computed with respect to the qualitative codes (PI, PSI, DI) assigned to information items. Figure 1 suggests two main trends. First, domain information (DI) was common for all task complexity levels, ranging from $91 \%$ for the remember task (simplest) to $42 \%$ for the create task (most complex). Second, problem information (PI) and problem-solving information (PSI) were more common for complex tasks, particularly for the most complex (create). In other words, the most complex task had more information items gathered to help the task doer understand the task requirements (PI) and gain strategies for solving it (PSI).

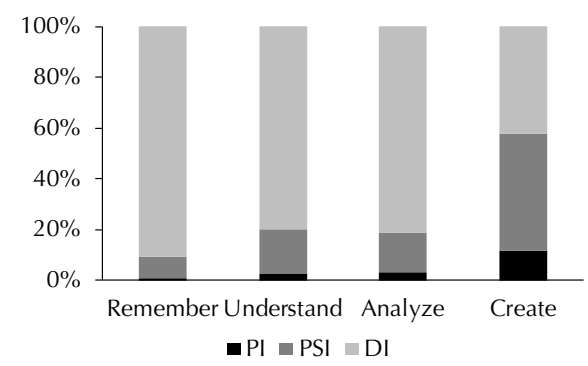

Figure 1: Task complexity effects on functional roles.

RQ2. Associations between info-types and functional roles: Figure 2 shows the mapped relations between info-types (facts, concepts, opinions, insights) and functional roles (PI, PSI, DI). Our RQ2 results suggest three main trends. First, facts, concepts, and opinions were mostly associated with DI. Second, information items coded as PI were mostly facts and concepts. One possible interpretation is that information that helps the task doer understand the task requirements (PI) tends to be objective and factual (facts and concepts). Finally, information items coded as PSI were mostly opinions and insights. One possible interpretation is that information that helps the task doer strategize on how to approach the task (PSI) tends to be subjective and experiential (opinion and insights).

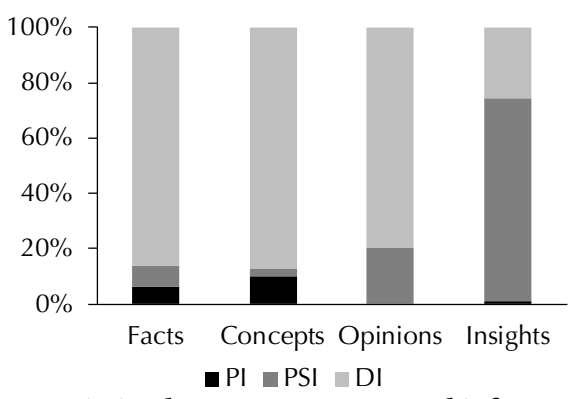

Figure 2: Association between statement and information types. 
RQ3. Effects of task complexity on the associations between info-types and functional roles: Figure 3 shows the effects of task complexity on the relations between info-types and functional roles. Our RQ3 results suggest one main trend. As task complexity increases, the relations between info-types and functional roles become more varied (i.e., less deterministic). Figure 3 shows several examples of this trend. For the remember, understand, and analyze tasks, facts mostly played the role of DI. Conversely, for the create task (most complex), facts played all three roles (PI, PSI, and DI). Additionally, for the remember, understand, and analyze tasks, PSI mostly originated from insights. Conversely, for the create task (most complex), PSI originated from all four info-types.

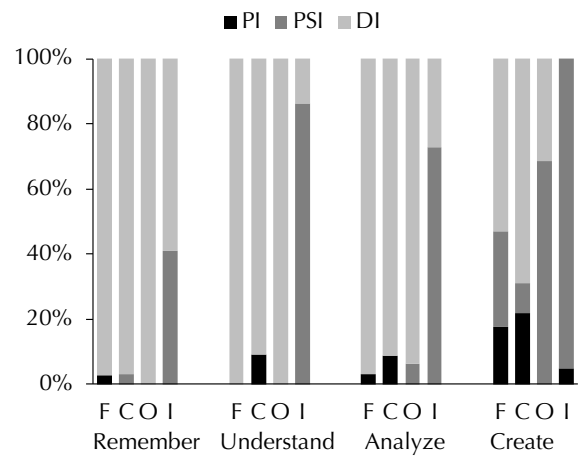

Figure 3: Task complexity, info-types, and functional roles

\section{DISCUSSION AND CONCLUSION}

In this section, we summarize our findings, compare them to those from prior work, and discuss their implications.

Cognitive task complexity affects the functional roles of task-related information: Our RQ1 results suggest that as task complexity increases, task-related information plays a greater role as PI (to help the task doer understand the task requirements) and PSI (to help the task doer strategize on how to approach and solve the task). This result is consistent with previous studies reported in Byström et al. [3, 5], in which complex tasks required more varied types of information and particularly more PSI.

Our research in this paper has two important differences compared to the studies from Byström et al. [3, 5]. First, while Byström et al. $[3,5]$ defined task complexity from the perspective of subjective a priori determinability, we defined task complexity from the perspective of objective cognitive complexity. Second, while Byström et al. [3, 5] analyzed genuine work tasks in a professional setting, we analyzed systematically designed search tasks in a lab setting. In this regard, our RQ1 results show that the trends observed in Byström et al. [3,5] can generalize to other definitions of task complexity and experimental settings.

A natural question is: Why do complex tasks require more PI and PSI? From the perspective of determinability, complex tasks have greater uncertainty about the task requirements. Thus, searchers may need more PI during complex tasks to gain a sufficient understanding of the task itself-to understand the task's requirements, scope, parameters, and basic underlying concepts. From Campbell's perspective [6], complex tasks have more paths to the outcomes and greater uncertainty about the paths. Thus, during complex tasks, more PSI may be needed to help the searcher decide which "path(s)" to follow and which to avoid.
Associations between info-types and functional roles: Our RQ2 results suggest a systematic relationship between inherent characteristics of information items and how they may be useful. All info-types had a tendency to serve as DI; objective and factual info-types (facts and concepts) had a greater tendency to serve as PI; and subjective and experiential info-types (opinions and insights) had a greater tendency to serve as PSI.

Our RQ2 results are also consistent with findings from Bystrom et al. $[3,5]$, in which complex tasks had greater use of humans as information sources. In our case, complex tasks had more information items coded as PSI (RQ1), and PSI had a strong tendency to originate from opinions/insights (RQ2). In other words, it appears that PSI (particularly useful during complex tasks) tends to originate from people's opinions, viewpoints, and experiences.

Cognitive complexity influences the associations between info-types and functional roles: Our RQ3 results suggest that as task complexity increases, info-types tend to play a wider range of functional roles. In other words, as task complexity increases, the associations between info-types and functional roles becomes less deterministic. For example, for our remember, understand, and analyze tasks, most PSI originated from insights. Conversely, for our create task (most complex), PSI also often originated from other info-types (facts, concepts, opinions). This trend suggests that during complex tasks, info-types tend to play more dynamic (vs. static) functional roles. Thus, searchers working on complex (vs. simple) tasks may benefit from more diverse info-types.

Implications: Our results have important implications for designing systems to support users with tasks of varying complexity. Our RQ1 results suggest that complex tasks require more PI and PSI than simple tasks. In other words, searchers working on complex tasks may need extra support in understanding the task itself (i.e., the task structure and requirements) and in deciding how to approach it. Prior work shows that cognitive task complexity influences search behaviors [7, 11, 12, 17]. Thus, future research should aim to develop systems that can infer a searcher's task complexity and provide more tailored support. For example, searchers working on complex tasks may benefit from seeing definitions of important task-related concepts (PI) and experiential information (e.g., blog posts) describing different approaches to the task (PSI).

Our RQ2 results found a few trends between info-types and functional roles. All info-types served as DI; objective and factual info-types (facts and concepts) served as PI; and subjective and experiential info-types (opinions and insights) served as PSI. This result suggests an opportunity to automatically identify task-relevant PI, PSI, and DI based on inherent characteristics of an information item. Prior NLP research on classifying statements as objective vs. subjective [16] may provide a starting point. Prior work has also used machine learning to classify information items as facts, opinions, and experiences [8].

Our RQ3 results suggest that task complexity influences the relations between info-types and functional roles. In general, we found that PSI mostly originates from insights. However, during our most complex task, all info-types played the role of PSI, which was most needed for the task. This trend also provides guidance for developing systems to support users. Specifically, during complex tasks, searchers may benefit from seeing different info-types.

Acknowledgement: Research supported by NSF grant IIS-1718295. 


\section{REFERENCES}

[1] Lorin W. Anderson and David R. Krathwohl. 2001. A taxonomy for learning, teaching, and assessing: A revision of Bloom's taxonomy of educational objectives.

[2] David J Bell and Ian Ruthven. 2004. Searcher's assessments of task complexity for web searching. In ECIR. Springer, 57-71.

[3] Katriina Byström. 1999. Task complexity, information types and information sources: examination of relationships. Tampere University Press.

[4] Katriina Byström. 2002. Information and information sources in tasks of varying complexity. FASIST 53, 7 (2002), 581-591.

[5] Katriina Byström and Kalervo Järvelin. 1995. Task complexity affects information seeking and use. IP\&M 31, 2 (1995), 191-213.

[6] Donald J Campbell. 1988. Task complexity: A review and analysis. Academy of management review 13, 1 (1988), 40-52.

[7] Robert Capra, Jaime Arguello, Anita Crescenzi, and Emily Vardell. 2015. Differences in the use of search assistance for tasks of varying complexity. In SIGIR. ACM, 23-32.

[8] Jorge Carrillo-de Albornoz, Ahmet Aker, Emina Kurtic, and Laura Plaza. 2019 Beyond opinion classification: Extracting facts, opinions and experiences from health forums. PLOS ONE 14 (2019).

[9] Bogeum Choi, Austin Ward, Yuan Li, Jaime Arguello, and Robert Capra. 2019. The Effects of Task Complexity on the Use of Different Types of Information in a
Search Assistance Tool. TOIS 38, 1 (2019).

[10] Anita Crescenzi, Yuan Li, Yinglong Zhang, and Rob Capra. 2019. Towards Better Support for Exploratory Search Through an Investigation of Notes-to-self and Notes-to-share. In SIGIR. ACM, 1093-1096.

[11] Bernard J Jansen, Danielle Booth, and Brian Smith. 2009. Using the taxonomy of cognitive learning to model online searching. IP\&M 45, 6 (2009), 643-663.

[12] Diane Kelly, Jaime Arguello, Ashlee Edwards, and Wan-ching Wu. 2015. Development and evaluation of search tasks for IIR experiments using a cognitive complexity framework. In ICTIR. ACM, 101-110.

[13] J. R. Landis and G. G. Koch. 1977. The Measurement of Observer Agreement for Categorical Data. Biometrics 33, 1 (1977), 159-174.

[14] Miamaria Saastamoinen, Sanna Kumpulainen, Pertti Vakkari, and Kalervo Järvelin. 2013. Task complexity affects information use: a questionnaire study in city administration. Information Research 19, 4 (2013).

[15] Robert S Taylor. 1991. Information use environments. Progress in communication sciences 10, 217 (1991), 55

[16] Janyce Wiebe, Theresa Wilson, Rebecca Bruce, Matthew Bell, and Melanie Martin. 2004. Learning Subjective Language. Computational Linguistics. 30, 3 (2004)

[17] Wan-Ching Wu, Diane Kelly, Ashlee Edwards, and Jaime Arguello. 2012. Grannies, tanning beds, tattoos and NASCAR: Evaluation of search tasks with varying levels of cognitive complexity. In IIiX'12. ACM, 254-257. 